\title{
A Review of Agent and Service-Oriented Concepts Applied to Intelligent Energy Systems
}

\author{
Pavel Vrba, Senior Member, IEEE, Vladimír Mařík, Senior Member, IEEE, \\ Pierluigi Siano, Senior Member, IEEE, Paulo Leitão, Senior Member, IEEE, Gulnara Zhabelova, \\ Valeriy Vyatkin, Senior Member, IEEE, and Thomas Strasser, Senior Member, IEEE
}

\begin{abstract}
The intention of this paper is to provide an overview of using agent and service-oriented technologies in intelligent energy systems. It focuses mainly on ongoing research and development activities related to smart grids. Key challenges as a result of the massive deployment of distributed energy resources are discussed, such as aggregation, supply-demand balancing, electricity markets, as well as fault handling and diagnostics. Concepts and technologies like multiagent systems or service-oriented architectures are able to deal with future requirements supporting a flexible, intelligent, and active power grid management. This work monitors major achievements in the field and provides a brief overview of large-scale smart grid projects using agent and service-oriented principles. In addition, future trends in the digitalization of power grids are discussed covering the deployment of resource constrained devices and appropriate communication protocols. The employment of ontologies ensuring semantic interoperability as well as the improvement of security issues related to smart grids is also discussed.
\end{abstract}

Index Terms-Active demand response, distributed generation, electrical grid, multiagent systems (MASs), power balancing, renewable energy sources (RESs), service-oriented architectures (SOAs), smart grid.

\section{INTRODUCTION AND MOTIVATION}

$\mathbf{T}$ HE CONVENTIONAL power grid is a vertically organized network, where the electricity generated by large power stations (i.e., bulk generators) is delivered over a

Manuscript received January 17, 2014; revised April 02, 2014; accepted May 12, 2014. Date of publication May 22, 2014; date of current version August 05, 2014. This research was supported by the Grant Agency of the Czech Technical University in Prague under Grant SGS12/188/OHK3/3T/13, and it received funding from the Austrian Climate and Energy Fund under project DG-EV-HIL (FFG 827987). Paper no. TII-14-0062.

P. Vrba and V. Mařík are with the Czech Institute for Informatics, Robotics, and Cybernetics, Czech Technical University in Prague, Prague 6, Czech Republic (e-mail: pavel.vrba@fel.cvut.cz; marik@labe.felk.cvut.cz).

P. Siano is with the Department of Industrial Engineering, University of Salerno, 84084 Fisciano, Italy (e-mail: psiano@unisa.it).

P. Leitão is with the Polytechnic Institute of Bragança, P-5301-857 Bragança, Portugal; and also with the Artificial Intelligence and Computer Science Laboratory (LIACC), 4169-007 Porto, Portugal (e-mail: pleitao@ipb.pt).

G. Zhabelova is with the Department of Computer Science, Computer, and Space Engineering, Luleå Tekniska Universitet, 97186 Luleå, Sweden (e-mail: gulnara.zhabelova@1tu.se).

V. Vyatkin is with the Department of Computer Science, Computer, and Space Engineering, Luleå Tekniska Universitet, 97187 Luleå, Sweden; and also with the Department of Electrical Engineering and Automation, Aalto University, FI-00076 Aalto/Helsinki, Finland (e-mail: vyatkin@ieee.org).

T. Strasser is with the Energy Department-Electric Energy Systems, Austrian Institute of Technology, 1210 Vienna, Austria (e-mail: thomas. strasser@ait.ac.at).

Color versions of one or more of the figures in this paper are available online at http://ieeexplore.ieee.org.

Digital Object Identifier 10.1109/TII.2014.2326411 high-voltage transmission system to the power distribution grid and finally to the customers. Today, this well-known energy landscape is undergoing dramatic changes caused by the largescale integration of distributed energy resources (DERs) into medium- and low-voltage power distribution grids [1], [2]. The upcoming increased usage of electric vehicles and smart home appliances imply a significant change in energy consumption patterns as well as in a way of temporal storing electricity. The volatile and stochastic nature of renewable energy sources (RESs) (mainly solar and wind) largely impact the balance of power generation and consumption and can in turn have an impact on the overall power grid stability and reliability [3], [4].

It is obvious that such challenges have to be addressed at two different levels. First, the electric power grid infrastructures have to be adopted to enable the smooth integration of DERs at a large scale. Second, the complementary automation software and communication infrastructures have to be modernized by extensive use of information and communication technologies (ICTs) to enable intelligent grid operation [5]-[7]. Today's ICT and automation solutions controlling power grids, primarily supervisory control and data acquisition (SCADA) systems, mimic their centralized and hierarchical-oriented structure [8]. They will soon reach their limits in terms of scalability, computational complexity, and communication [9].

It is inevitable that future smart grids will become a distributed ecosystem with thousands and even millions of heterogeneous components with local intelligence interacting on both the electricity and information level. Decentralized control architectures with distributed decision finding offer more flexibility, extensibility, and scalability. Open and service-oriented architecture (SOA), adequately responding to the challenges of the grid's decentralization [10] but standardized solutions are especially required to guarantee interoperability [11], [12].

New methods have to implement multicriteria optimizations, where the local interests and constraints of various actors have to be aligned with the global system's objectives (i.e., stability, quality, and security of the power supply). Such empowerment with the digital layer is denoted as a smart grid [3], [4], [13]. A key research question today is how to make the power grids "smarter" and the components more intelligent? It is likely that modeling and design techniques based on artificial intelligence have to be exploited in order to create entities that are able to sense and act in the environment, gather and reason knowledge/ information, learn and adapt, intentionally plan own activities, and communicate with others. 
Several software architectures have been proposed to address the challenges of developing systems with highly decentralized intelligence. One of the most relevant technologies is multiagent systems (MASs) [14], [15]. Their potential in industrial domains has already been documented in several simulation studies, pilot projects as well as real-life applications [16], [17]. As discussed in this review, MAS-based approaches receive a significant attention as a proper automation technology also in the field of smart grids [18]-[22].

Besides MAS, another promising approach managing distributed systems is provided by the SOA concept. It also provides a solid foundation for building smart grid applications. SOA uses similar foundation principles as provided by MAS; composite applications consisting of loosely coupled entities which communicate via messaging in a networked environment [23]. A common way of developing SOA-based solutions is using web-service standards, such as web-service description language (WSDL), simple object access protocol (SOAP), or universal description, discovery, and integration (UDDI). They have gained a broad industry acceptance [24] and therefore are suited also for the domain of smart grids [25], [26].

In both cases, smart grid components such as distributed generators (DGs), intelligent switches, smart transformers, sensors, electric appliances, or electric vehicles can be monitored and controlled by an autonomous software component - being it either an agent in the MAS sense or a service in the SOA sense. These units interact and coordinate their activities in order to achieve the desired behavior such as the balance of power generation and consumption [27], [28].

This paper provides an overview of research results using MAS and SOA principles in smart grids. Its main goal is to discuss important MAS/SOA features used in the power systems domain and to provide a review of important projects, activities, and achievements in the domain. Future research directions are sketched out.

In this review paper, Section II identifies key challenges faced by future power systems including distributed generation, load balancing, energy markets, and fault handling. Most important $\mathrm{MAS} / \mathrm{SOA}$ features supporting future smart grid operation are discussed in Section III followed by an overview of important projects in Section IV. Section V concludes this review outlining future trends and research activities.

\section{Challenges of Future Power Distribution Grids}

\section{A. Large-Scale Integration of Renewables and Storages}

The integration of DER in the European electricity network is strongly supported by the European Union (EU) energy policy. It is expected that in 2020, the total contribution of DER-based generation could go beyond $20 \%$ [29]. The key driver is the "Energy and climate change package" of the European Commission from 2007 that declares the energy targets for EU-20\% reduction of greenhouse gas emissions, $20 \%$ of RESs, and $20 \%$ of the overall energy consumption reduction by year 2020 . Germany sets even more ambitious targets - $35 \%$ of renewable energy till 2030 and even $80 \%$ till 2050. The pace of DER deployment is however still slow, facing the issues related to

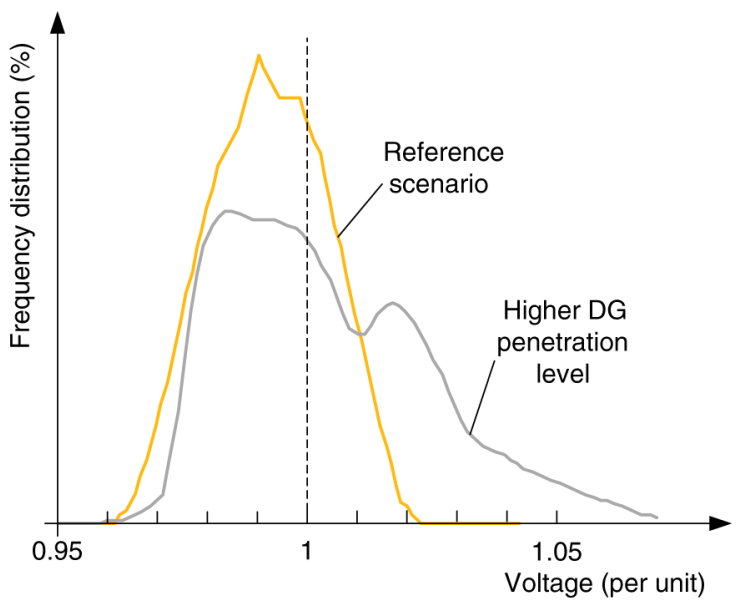

Fig. 1. Impact of DG on the frequency distribution of voltages in the grid [39].

technology, market, and regulation [30]. Similar goals and activities are also observed in other parts of the world (North America, Asia, or Australia) [31].

DERs are energy sources usually connected to the power grid at medium- or low-voltage levels. A large subcategory of DERs is dedicated to RESs such as solar, wind, biomass, and hydro, and these two terms are often interchanged. Devices used to store energy (batteries, super capacitors, or flywheels) have to be included as well because they play a critical role in demandsupply balancing [32]. Also electric transportation using (plugin) hybrids and electric vehicles will play an important role in the future [33], [34].

A major benefit of having the energy sources dispersed over the grid is the local generation possibility, close to where it is consumed. Thus, there is no need to transport it over long distances and suffer from energy losses. Another obvious benefit of using renewable sources is their significant contribution to the reduction of greenhouse gas emissions. On the other hand, high penetration of RES stresses the whole power system in terms of voltage or frequency fluctuations caused by the intermittent and random nature of solar and wind sources. Critical situations occur when voltage or frequency exceeding given limits [35]-[38]. For example, Fig. 1 provides a qualitative example about the frequency distribution of voltages measured at a specific node in the power grid with different amount of distributed power generation. A more detailed analysis of the impact, challenges as well as opportunities integrating distributed generation into power grids is provided in [39] and [40]. In order to cope with such unwanted effects in the power grid the following strategies can be applied.

1) Intelligent Grid Operation Using Advanced ICT Solutions: A way to manage grid stability and power quality issues is related to how the power system is operated. Current approaches are rather passive - the DERs are connected to the grid in a fit-and-forget way, either relying on a sufficient capacity to absorb the over production or directly putting constraints on DG developers in terms of maximal capacity that can be connected in particular grid segment. This however goes against the efforts to maximize the renewable power generation. However, active and intelligent management principles have to be applied to enable efficient integration of DER [36], [39], 


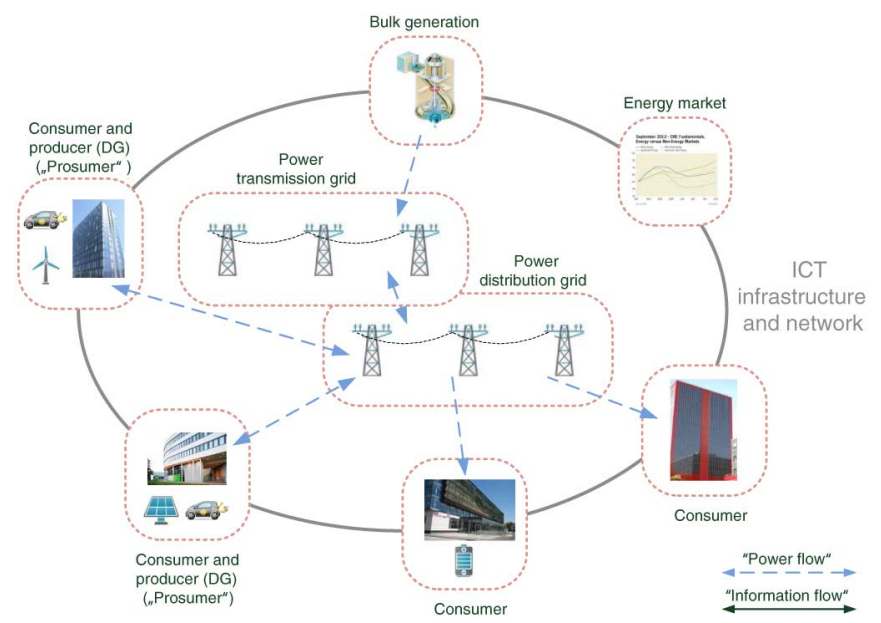

Fig. 2. Smart grid vision-intelligent integration of all stakeholders.

[41]. They will require employing active elements into the network with embedded intelligence and two-way communication capabilities [5]-[7]. The intelligence of the elements can be defined as the ability of gathering and processing knowledge, autonomous decision making, as well as communication and coordination of activities with others. Accompanying factor is the context-awareness as the ability to sense and react in a real environment. Fig. 2 provides an overview of such a smart grid with advanced functions through the usage of ICT technologies and approaches.

2) Aggregation of DERs: Another important technique to mitigate the misbalancing effects of distributed generation is the aggregation of DERs together with flexible loads and energy storage capacities into larger units that act as a single entity on the energy market. There are various names/terms used for such clusters of generators and consumers like virtual power plants (VPP) [42], micro grids [43], or grid cells [38]. The added value of such an aggregated entity is a better position on the energy market, local demand-supply balancing and reduction of peak loads. This cluster can also provide power balancing as an additional service to the main power grid. The micro grid is coupled with the main grid through a single point and thus can be easily disconnected in order to work autonomously in islanded mode [21], [44]-[46]. This feature is useful when a critical fault is threatening the main grid - the micro grid can quickly disconnect from the main grid to avoid being impacted by the failure and to contribute to further spreading of the fault. During islanded mode, however, the micro grid has to rely on its own generation only and thus electricity is first delivered to critical consumers. Major issues of VPPs are (partly) missing legal and contractual frameworks (depending usually on national grid codes and interconnection rules) hindering the wider exploitation of this concept.

3) Control Strategies for Grid Optimization: Another widely discussed approach is voltage control in rural power distribution grids to maintain power quality through fluctuating distributed generation [36], [37], [47]. The principle is an intelligent control of on-load tap changing (OLTC) transformers that can adjust the voltage level by setting the transformer's windings to a new transmission ratio. In a traditional passive approach, each OLTC monitors the local voltage level and if it exceeds a fixed set point, a tap change is applied to compensate the deviation. In an active approach, the real-time voltage measurements taken at multiple critical nodes of the network are used to operate the OLTCs. Even more complex solutions using the coordination of the OLTC operations voltage control possibilities provided by DGs and storage systems connected in the same area [48]. Sometimes also regulators are used to decouple voltages in parts of the network in which the levels are different. Typically, it is applied where a DG in placed in one feeder and a load in another one [37].

4) Demand Side Operations: A very promising technique for mitigating fluctuations caused by renewable sources is the usage of electricity storage systems used in plug-in hybrids and electric vehicles. They could participate in the so-called vehicle-to-grid power transactions as they represent controllable charges that can absorb electricity and provide it back to the grid when needed [49], [50]. Considering Germany's target to have 1 million electric vehicles by 2020 , it constitutes a massive capacity that can provide enough electricity for $7 \mathrm{~min}$ during the day or even 30 min over the night to the whole country. First attempts to design conceptual frameworks integrating electric vehicles into power grids have already been reported in the literature [33], [34].

5) Cost Assessment: Benefits and costs have to be quantified in order to achieve the acceptance of new grid solutions with a high share of DERs by all stakeholders (including regulators, utilities, and customers) [36]. The study in [37] presents a method analyzing economic questions due to the reinforcement of power grids which is needed to connect certain DG capacities. It also gives quantifiable estimations of extra connectable DG capacity while preserving the current network and maintaining the level of quality of supply. The results show that the application of active approaches such as coordinated voltage control and decoupling is economically competitive with respect to conventional grid reinforcement methods.

\section{B. Energy Markets and Trading}

Together with changes required at the technical also changes at the market level are necessary. Today's electricity trading takes place only at higher levels of the power grid hierarchy, involving mainly large generators and consumers. Smaller aggregated units can also participate, but typically restriction on the minimum size of a power generation unit exists (often depending on national rules). A mechanism called balancing responsibility in which the market participants referred to as balancing responsible parties, are obliged to provide daily plans for generation, transportation and consumption. These plans with a typical resolution of 15 or $30 \mathrm{~min}$ have to be handed over to transmission system operators (TSOs) the day before. The purpose is to force the players to stick with the plans in order to ensure stability of the grid. If an actor deviates from the plan he is charged for the imbalance. TSOs continuously monitoring the power grid states on a second basis and adjusting the contracted reserve power plants up or down to mitigate any occurring imbalances [41].

1) Market Integration of DER: With the increasing usage of DERs the trading has to penetrate also the lower levels of the power system. New business models for generation, consumption, and balancing are needed to enable thousands, potentially millions of smaller participants, being it DERs, 
clusters of residential buildings, or even single residential homes to participate actively on the energy market [10], [51]-[53].

2) Electronic Markets: There are efforts to establish electronic energy markets where generators and consumers actively participate in buying and selling electricity. In contrast to current wholesale market operation in a day-ahead regime, the prices in the suggested model are established in a real-time fashion reflecting actual needs and conditions in the grid. Obviously, prices go down if there is actual over-production of electricity and increase on the other hand in peak periods. A distribution company acquisition market model is presented in [54]. DG units and customers (with load curtailment options) represent new agents that actively participate on the market.

3) Prosumer Role and Real-Time Reaction: In the new concept, the market participant can play multiple roles. The residential area, which is typically a consumer, can be at the same time the energy producer (having photovoltaic panels on the roof) or can provide energy storage capacities (having an electric vehicle in the garage). A consumer that can act also as a producer is referred to as prosumer [55] (see Fig. 2).

The effort of each market participant is to optimize its energy consumption and/or production profile. This leads to shifting the consumption to lower price periods and the production to higher price periods. By such a mechanism of a real-time market the demand-supply balancing is naturally achieved [10]. The realtime energy pricing helps to better manage fluctuations of both, power supply from renewable sources, and energy demands of residential and commercial customers. The varying prices of electricity over a day are also referred to as smart tariffs, dynamic tariffs, or real-time price signals.

4) Local Trading: Exploitation of electronic energy markets at the lower hierarchy levels gives the opportunity of local electricity trading within micro grids [10], [30], [53]. The over-production of electricity supplied by a particular actor in a cell [combined heat and power (CHP) units], could be offered to other actors like heat pumps or local electricity storage in a residential home. Instead of selling the energy at low prices by former actor and buying the expensive energy from utilities by the latter one, they can make a direct local contract with dynamic negotiated prices, which will be mutually beneficial for both of them. It has to be noted that (regional/national) grid codes and interconnection rules have to be fulfilled. As a consequence, maybe not all technical possibilities are allowed.

\section{Fault Handling Strategies and Approaches}

Massive penetration of distributed generation from renewables together with increasing energy consumption implies higher risks of power quality drops, failures, and outages. The most serious and harmful are cascading failures that can lead to a total system collapse-a blackout. Examples are the Northeast blackout in the United States in 2003 affecting 50 million people or the latest massive blackout in India in 2012 leaving over 700 million people without electricity [56].

The reasons for such failures are typically grid overloads followed by a disconnection of whole feeders and/or DGs. This causes an overload in other parts of the system influencing also other feeders and components. Very common is the situation in which the lines, carrying excess current, get hot and thus lengthen and sag between the towers. If they sag too much a flashover to nearby objects (trees, etc.) can happen causing a transient increase in the current. An overload protection system detects the high current and quickly disconnects the faulted feeder/line from service. The flow of the excess current has to be shifted to other transmission lines. If they also run near or at their capacity their protection will also trip causing a cascading failure effect. As a further consequence, also power plants are disconnected to prevent damaging the generators [57].

1) Outage Management: When the failure happens, it is of vital importance to localize the fault as soon as possible and restore the power. In this task, the control room operators are assisted by outage management system (OMS) - the ICT system equipped with a detailed power grid model and rule-based engine to detect the fault location. However, it is still likely that the system operators rely on outage report calls from customers, which have to be manually entered into the OMS [58].

2) Fault Identification and Localization: More advanced solutions make use of fault passage indicators (FPI) that are installed at strategic points in the power grid and report the fault conditions to the control room automatically (e.g., over mobile phone network using GSM/GPRS). It is suggested that remote FPIs can provide additional value-added services in power grids with high degree of distributed generation such as real-time reporting of load flows and flow direction [59]. Complementarily to FPIs, Smart Meters installed at customer premises also provide outage detection and notification capability. They can transmit real-time outage alerts to utilities and verify whether the power has been restored or not [60].

3) Fault Prevention: Fault prevention and mitigation techniques are also intensively studied. One of the most discussed approaches to mitigate the effects of a threatening fault or blackout is to disconnect parts of a power grid and to form a local island in order to avoid the fault propagation [45]. In this mode, the remaining part of the network operates autonomously and therefore separated from the rest of the power system. Techniques of intelligent load shedding should be applied during islanding to switch off noncritical appliances and preserve power for vital loads [30], [61]. By partitioning the grid into multiple separated and autonomously acting islands, effects of failure propagation might be considerably minimized. When the source of the fault is removed, the islands can reconnect to the main grid, successively in a coordinated manner to restore the normal operating state.

4) Self-Healing: Such a smart grid capability is also widely discussed. It is a collection of techniques for early detection of threatening failures and advanced control approaches to take preventive actions [62]. An agent-based system for self-healing capabilities is presented in [63]. There are special prevention control agents that are in charge of forecasting states that could lead to failures. Issicaba et al. [44] describe an agent-based architecture for islanding and self-healing of active distribution grids. They consider segmenting the grid into blocks controlled by agents which cooperatively prepare plans of actions isolating the faulty parts from the grid. Another agent-based approach for fault detection and reconfiguration used in the smart grid context is presented in [64]. The agents calculate the optimal network configuration in terms of identifying which switching devices 
should be opened or closed in order to minimize power losses in the power distribution grid.

\section{Benefits AND Challenges of Industrial Adoption of MAS AND SOA IN POWER System AUtOMATION}

\section{A. Brief Overview of MAS and SOA Principles}

MAS is a paradigm that allows to design large-scale distributed control systems in an alternative way. It is derived from the field of distributed, artificial intelligence, and uses autonomous and cooperative agents, exhibiting modularity, flexibility, and robustness [65], [66]. This concept exhibits the capability to distribute the control over a network of software entities, the agents. Each one has its own knowledge, skills, and autonomous proactive behavior. They cooperate together achieving global objectives and offering a decentralized control structure, opposite to traditionally used rigid and centralized solution. MAS technology provides a fast response to condition changes and supports reconfigurability [66].

SOA is based on providing and requesting services. A service is usually a software entity which encapsulates business or control logic as well as functionalities provides by a component (which can be internally represented by an agent). Enterprise service bus (ESB) is a SOA framework that can be used as a backbone for supporting the interoperability among distributed (agent-based) systems. Typically desirable capabilities of ESBs include process orchestration, protocol translation, hot deployment, versioning, lifecycle management, and security, supporting the IT-vertical integration.

Both approaches can be combined together in terms of embedding the intelligence, possessed by agents, into webservices, which are broadly accepted by industry supporting interoperability of systems and components [67]. An overview of the integration of the MAS and SOA principles as well as the usage of web-services in this context is discussed for example by Guo et al. [68] and Herrera et al. [69].

\section{B. Benefits}

Smart grid can be seen as a complex and large-scale system of systems. It has usually a high number of nodes (generators and transformers) and interconnections (distribution lines and communication links). The IEC Strategic Group 3 (SG3) defines such nodes in their smart grid conceptual model as hardware/software resources that can be of different types, namely the power generation (including solar and wind energy plants), distribution, substations, and users (i.e., residential, commercial shops, and industry) [12]. Moreover, also control components for energy management (e.g., metering infrastructure and data handling) and SCADA systems are mentioned. The possibility to have consumers that are able to locally generate power and feed it into the grid as well as electric vehicles that can charge their batteries at any time and serve as energy storage, increases the complexity of the whole system [13].

One of the most important challenges in the smart grid context is the employment of the necessary ICT infrastructures. It will help to manage and operate such a complex system as well as to transform the existing vertically and centrally/hierarchically structured topology into a more decentralized smart grid. All these factors show that the future system becomes a complex and large-scale cyber-physical system [25]. Thousands or even millions of autonomous, interacting components are connected whose functionalities have to be monitored and coordinated in real-time or near real-time. Additionally, as stated in [70], this ICT apparatus will give the consumers and producers a full control of their consumption and generation and will enable to implement advanced demand-response services such as peak shaving or load shifting.

A proper software technique that would fulfill and cover the above discussed requirements is the MAS approach. Its potential for deployment in the industrial domain has already been proven in several simulation studies, pilot deployments as well as reallife applications [15]-[17]. With MAS technology important features required by smart grids can be realized. A decentralized ICT-based control approach can be realized supporting the following important features [71].

1) Modularity: Allows adding new elements (e.g., DER and consumer) to the power system in a plug and play manner (without the need to stop, reconfigure/reparameterize, or reprogram and restart the whole system or parts of it).

2) Scalability: The whole system can be extended/enlarged in a simple way facing the (future) large-scale integration of energy resources (e.g., DER and electric vehicles).

3) Reconfigurability: Changes can be performed on the fly (e.g., an agent can stop, modify its behavior or strategy and start again without affecting the other components of the system). It should target hardware- and softwarerelated topics (e.g., grid topology and DER functions).

4) Robustness: Losing one agent does not imply a system failure (e.g., if an agent associated with a customer fails and the system continues to run).

Using MAS-based principles and concepts, each component in the smart grid can be monitored and controlled by an autonomous software component, the agent. Agents are interacting according to proper coordination logic, to achieve the system goals, by combining their individual skills and knowledge, as illustrated in Fig. 3. The employment of MAS approaches enhance several functionalities inherent to smart grid, such as distributed and real-time monitoring, diagnosis, self-healing, self-maintenance, and negotiation (e.g., supporting dynamic clustering formation, price evolution, and grid management). Together with SOA-based principles a flexible, interoperable, and scalable ICT/automation solution can be realized. The achievement of these functionalities requires the design and implementation of proper coordination mechanisms and also suitable adaptive algorithms to be embedded in the distributed agents. Of particular importance is the consideration of biologyinspired methods, such as self-organization, to enhance MAS/ SOA-based solutions with more powerful and dynamic mechanisms in the power systems domain [15].

\section{Challenges}

However, the adoption and wide usage of MAS/SOA-based automation and control solutions in the power systems domain is quite slow, despite the great number of research results achieved [72]. There are several reasons hindering a large-scale roll-out of 


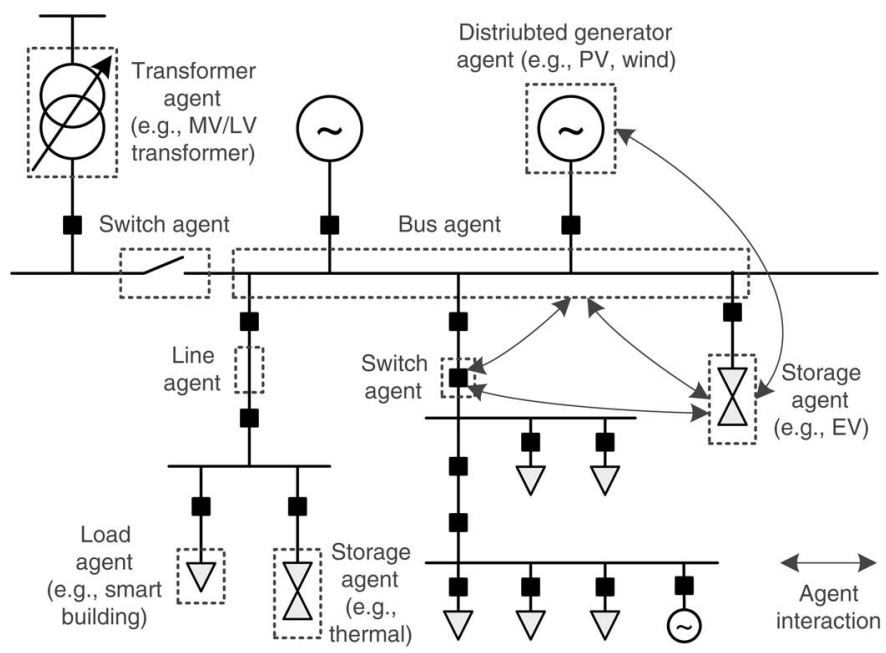

Fig. 3. Modeling a smart grid using multiagent technology [71].

smart grid technology. One of the most important issues is the necessary huge amount of investments on all levels of the existing power system infrastructure to transform it into a real smart grid. Therefore, the implementation of proper solutions is an evolutionary process rather than a revolution [3]. However, due to the massive installation and integration of dispersed, fluctuating generation units more intelligent and distributed concepts as well as architectures are required on a long term basis. Beside the slow adaptation of smart grid concepts in the field also some shortcomings related to MAS/SOA have to be solved at first. The following reasons can be attributed for their uptake.

1) Emergent behavior: due to the nature of the technology, the designed system can exhibit a wide variety of expected but also unintended behaviors, which may lead to inefficient or unstable operation [15], [17].

2) Design methodologies and architectures: there is a lack of mature and well accepted design methodologies and standardized agent architectures appropriate for smart grids [73]. Being expert in power systems automation rather than agent technology, the researchers often focus on the decision making algorithms rather than the agent architecture, considering agents as an intelligent actors by definition [73]-[75]. Although some of them have proposed specific design methodologies [76], others just report on "one-off" development processes for a dedicated system [75]-[78].

3) Interoperability and IT vertical integration: the interoperability of agent-based system should be ensured at both, the syntactic and semantic level. The former one should achieved by employment of common agent communication languages such as Foundation for Intelligent Physical Agents (FIPA)-ACL [79], while the latter one will rely on the definition of proper ontologies for the power systems domain. The vertical integration issue also arises when trying to integrate MAS into complete engineering solutions. SOA concepts and existing implementation such as web-services, RESTFul, or ESB concepts are expected to help solving the integration issues as they are becoming widely accepted in the industrial environments. MAS as well as SOA approaches can be advantageously combined together [67], however, a common approach is necessary for its adoption in the domain of smart grids.

4) Execution on field devices: the agents will be executing (running) on field devices, such as intelligent electronics devices (IEDs), DERs, PEVs and others. It is not clear up to now from the related literature, how to implement widely used Java-based agents on such devices addressing the related deployment challenges.

Together with the growing experience in real-life deployments, the maturity of MAS/SOA technology addressing these challenges will crystallize. The systematic application of existing and new agent-based architectures and SOA design methodologies will define suitable ones for the power system automation domain and will help to improve them, by addressing practical application issues.

At this stage, there is a need to facilitate the use of agent technology for practical applications in the power system automation domain. The research needs to be directed at defining suitable agent architectures, methodologies, and tools that are specific to the power system automation domain and that are aimed at the domain engineer. This will enable a domain engineer to develop agent-based solutions for smart grid automation domain problems.

\section{Related Work AND PRojects}

The purpose of this section is to provide an overview of major research activities focusing on the application of modern software technologies in the field of intelligent energy grids. The main attention is paid to those solutions that exploit principles of designing software in form of interoperable, loosely coupled and reusable entities, especially based on MAS/SOA. It is out of scope of this review paper to cover also other approaches and ICT concepts/technologies applied in the smart grid context as it is covered for example by Cecati et al. [5].

\section{A. Distributed ICT Infrastructure-Distributed Intelligence in CRitical Infrastructures for Sustainable Power (CRISP)}

The CRISP project, ${ }^{1}$ which finished in 2006 , was aimed at developing a distributed intelligent ICT infrastructure for sustainable power systems. It proposes a concept of Grid Cells, in which the network is divided into subnetworks independently managed by software agents called smart grid automation device (SGAD). It is responsible for making the local decision regarding the current and estimated production/consumption, control, fault detection, and reconfiguration. The grid agents can communicate with each other about the allocation of resources as well as with the higher control levels of the grid where the information is aggregated and processed [38].

This project declares the development of an agent-oriented fault handling and reconfiguration solution. There is a single agent called "Help Tool for Fault Diagnosis" (HTFD), which collects data from grid cell devices, especially from FPIs. After a signal from FPI is received the HFTD agent locates the faulty section and proposes fault isolation steps to restore the fault.

${ }^{1}$ Available: http://www.crisp.ecn.nl 
Tests were performed on a simple cell composed of two interconnected medium-voltage feeders and standard PC computers communicating over Ethernet using a simple transmission control protocol (TCP)-based protocol. The achieved results show that the fault can be correctly isolated in less than $1 \mathrm{~min}$, even if the communication speed is low (10 Kbit/s) [38].

\section{B. Power Balancing With PowerMatcher}

One of the results of the CRISP project is the PowerMatcher ${ }^{2}$ tool used for balancing power demand and supply in electrical networks by use of agent-based electronic markets [10]. Each component is represented by an agent, which tries to control the device's energy generation and/or consumption in the most economical and optimal way. The energy produced and consumed by the device is sold and bought by these device agents on the market, organized into a logical tree. Leafs are the logical device agents representing DER devices. The root of the tree is the auctioneer agent who is responsible for the equilibrium price forming process. In between, there can be concentrator agents as representatives of device agent clusters. The process of price negotiations is synchronized in the whole tree by triggering the execution event by the root auctioneer agent. This is a signal for concentrator agents to collect the bids from lower levels and send the aggregated information back upwards. The auctioneer then determines the equilibrium price, which is communicated downward back to the device agents. On the basis of market price and its bid function, each device agent can determine the power allocated to the device. There are different categories of device agents with different bidding strategies aimed at economic optimization of local processes within given constraints, such as gas generators, storage facilities, and CHP units. The natural effect of self-interested behavior is shifting the consumption to low-electricity price periods and the production to high-cost price periods [41].

There are two field tests described in [41] that document the benefits of application of the PowerMatcher technology. The first test is related to commercial portfolio balancing showing the reduction of the grid imbalance by $40 \%$ to $43 \%$. The second test carried out in the Netherlands was related to the aggregation of micro-CHP into a VPP. It documents a peak load reduction of $30 \%$ in summer (field test) and 50\% in winter (simulation).

\section{Microgrids and More Microgrids Projects}

More Microgrids (follow-up of the Microgrids project) was aimed at developing a concept of small-scale electricity grids operating at the low-voltage level (see Section II-A). The goal of the project was to investigate the applicability of MAS concepts to control small power grids on islands [30]. The core component of this approach is an intelligent load controller (ILC), which is able to communicate with houses over power line in order to control their loads in an intelligent manner. One of these devices is for example a water pump, which is considered to be of low importance and thus can be switched off by the ILC in case of power shortage. As second device, a power socket and any load connected to it was considered.

\footnotetext{
${ }^{2}$ Available: http://www.powermatcher.net
}

The ILC is an embedded computer (Intel Xscale processor at $400 \mathrm{MHz}, 64 \mathrm{MB}$ RAM) with Windows CE 5.0, which is installed at the point of common coupling before the energy meter at each house. The ILC is able to take and process measurements including voltage, frequency, and current in order to report it to the micro grid controller. This task is carried out by an intelligent agent [based on the Java Agent Development Framework (JADE) 3 agent platform], embedded in ILC, which communicates with the so-called microgrid central controller (MGCC) agent. This entity collects data from all the grid devices, including photovoltaic panels, a battery bank as well as a diesel generator. Its task is to balance consumption and production of electricity in the micro grid with the main goal to avoid situations in which the diesel generator has to be started.

In general, if there is a surplus of solar energy produced, the MGCC agent sends a request to battery bank agent to consider the charging option. On the other hand, if the energy demand is greater than the production units can cover, the MGCC agent sends a message to all ILC agents that a load shedding is needed. The ILC agents are able to negotiate among themselves which of the nonimportant loads can be disconnected from the grid, while respecting the house residents' preferences regarding the living comfort.

Field tests were carried out on Greece's Kythnos Island, with an experimental grid containing 12 buildings, a $10-\mathrm{kW}$ photovoltaic system, 53-kWh battery banks, and a diesel generator. The main goal of the experiment was to test the MAS-based control in a real-world setting. As reported in [43], the negotiations between the agents about optimal shedding of irrigation pump loads was based on the english auction using an ontology derived from the IEC 61970 standard, the common information model (CIM) for energy management.

Within the same project, the exploitation of MAS controlling a VPP was investigated [42]. The VPP concept covers the aggregation of various production units, such as photovoltaic panels, CHP units, or wind turbines that act as a single entity on the energy market. Also, loads can be covered by such a VPP. In the proposed solution, each unit is obviously associated with an agent, which ensures its control in coordination with other agents. The agents belonging to the units in the same physical area are grouped into single a MAS, represented by the management agent being able to negotiate with others on the electronic market. This solution was also developed using JADE agent platform in conformance with the FIPA standard [79].

\section{Business Solutions for DER-EU-DEEP Approach}

The goal of the EU-DEEP ${ }^{4}$ project was to design the methodology for producing business solutions for enhanced DER deployment in Europe by 2010. The project identified main technical and nontechnical barriers preventing wider deployment of DER across Europe and derived a set of recommendations and directions to ensure efficient and sustainable integration of DER in the current electrical power system. The project also focused on new business models and trading strategies, in which the end

\footnotetext{
${ }^{3}$ Available: http://jade.tilab.com

${ }^{4}$ Available: http://www.eu-deep.com
} 
customers, aggregated to larger units, play an active role on the market [51].

Numerous field tests were carried out within EU-DEEP. One of them was focused on the technical feasibility of the MASbased decentralized control architecture for aggregation of generation and loads. The agent technology is used to implement the concept of "fair" load shedding, in which the agents, controlling loads and production units, negotiate on which loads should be turned off according to the total power available [30].

\section{E. Distributed Control of DER-INTEGRAL}

The objective of the European project INTEGRAL ${ }^{5}$ was the development of a platform for distributed control and coordination of aggregated DERs based on industrial ICT. In three field demonstrations normal, critical and emergency scenarios have been covered [53]. The first scenario deals with a cluster of residential houses equipped with micro-CHPs, heat storage tanks, intelligent thermostats and switchable loads. The residential houses are aggregated into a VPP that interacts with the rest of the grid in terms of buying and selling energy on the market to achieve optimal demand-supply matching. The implementation leverages the PowerMatcher agent-based technology originally developed in the CRISP project (see Section IV-A). The field tests are obviously identical with those mentioned in Section IV-B for the PowerMatcher approach. In the second scenario, the micro grid running in islanded mode consisting of a number of DGs, a backup power diesel, and shiftable loads related to domestic consumption like heating and cooling. The agent-based supply-demand coordination is applied again to react to critical situations in the power grid. It is performed by local controller agents associated with real field devices and the MGCC agent responsible for overall coordination of the system. The agents are implemented in JADE and ZigBee technology is used to transfer data from field devices to the central control system. Apparently, the solution bears considerable similarities to the MoreMicrogrids project (see Section IV-C), including the same name of the central controller agent, without explicitly mentioning how these two projects are related and in which the solution has been originally developed.

\section{F. Aggregation of Smart Houses (SmartHouse/SmartGrid)}

SmartHouse/SmartGrid ${ }^{6}$ is another European project aiming at delivering the ICT-based solution for technical and commercial aggregation of smart houses and their integration into the grid. The approach is based on the following elements: 1) inhouse energy management system considering user feedback, real-time tariffs and smart appliances; 2) software architecture based on agent technology for aggregation of houses to clusters; and 3) use of SOA technology (i.e., web-services) for the interaction of the smart house with other entities in a smart grid. Even in this project, as in CRISP and INTEGRAL, the PowerMatcher agent software is used.

The control for the micro grid is also based on agent technology. The Magic MAS is designed to allow controlling each unit such

\footnotetext{
${ }^{5}$ Available: $\mathrm{http} / /$ integral-eu.com

${ }^{6}$ Available: http://www.smarthouse-smartgrid.eu
}

as a battery bank, a wind turbine, or a controllable load by an individual agent. The agent system, after negotiation with suppliers, receives a schedule for power production and consumption including price information. Afterward, the agents decide how to implement the schedule [26].

\section{G. Smart Grid Control With Real-Time Prices-EcoGrid}

One of the latest initiatives in the field of smart grids is the European EcoGrid ${ }^{7}$ project. The main objective is the full scale demonstration of a smart grid solution with a mix of DER, including more than $50 \%$ of renewable sources. The demonstration will take place on the Danish Bornholm Island, where the majority of 2000 residential consumers will be equipped with intelligent controllers enabling them to be responsive to variable energy prices. The price signals will be updated every $5 \mathrm{~min}$ to reflect the needs for up- or down-regulation to mitigate the system imbalance. According to [52], the real-time energy market will be implemented by using state-of-the-art ICT technologies, leveraging some of the proven technologies like the PowerMatcher approach.

\section{H. Single Agents in Smart Grids}

This project was focused on the development of an infrastructure for intelligent control of cooling and heating in buildings within a smart grids environment. The objective is to optimize electricity consumption by precooling during low-cost periods. In countries with hot weather, like Greece, where field tests of this project were performed, or in India, where the massive blackout happened in 2012, the air-conditioning is the main cause of peak demands and can lead to serious problems especially during extremely hot periods [79].

The single agent thermal model predictive controller (SAT-MPC) was developed to estimate the future indoor temperature as well as the power consumption. The goal is to predict the optimal point in time to start the precooling in advance of the predicted peak period, while keeping the indoor temperature comfort for the inhabitants. The thermal model, implemented in LabVIEW, was quite complex, considering heat fluxes of inner and outer walls, absorbed solar radiation, heat losses from windows and doors, and inner heat sources.

Although referring to "agent" in the paper title as well as in the name of the developed model predictive controller it does not seem that a true MAS approach was utilized.

\section{Active Demand Concept (ADDRESS)}

The objective of the ADDRESS ${ }^{8}$ project is to develop a commercial and technical framework of the so-called active demand, which means active participation of domestic and small commercial consumers in the electricity market [81]. The architecture exploits the concept of aggregation of demand flexibility provided by consumers and prosumers to form services offered to the electricity market participants. Overall, 31 services have been identified such as power flow control, smart load shedding, balancing of generation and consumption, and reserve capacity. The aggregation function is ensured by the aggregator entity,

\footnotetext{
${ }^{7}$ Available: http://www.eu-ecogrid.net

${ }^{8}$ Available: http://www.addressfp7.org
} 
which is expected to master up to 100000 consumers. The aggregator is able to forecast the short- and long-term consumption-related behavior of the aggregated group and on the basis of market price estimation, it broadcasts the price and volume signals to consumers as incentive to adjust the consumption.

The communication infrastructure is designed according to SOA concepts through the use of IEC standards for web-service implementation (i.e., IEC61968-1-1 and IEC61968-1-2) [12]. The content of XML messages sent among services is encoded using the IEC CIM for smart grids according to IEC 61968 and IEC 61970. There were two simulation platform prototypes developed - one based on the Apache AXIS web-service framework and the second one utilizing OpenESB and BPEL service orchestration [82].

\section{J. Agents for Smart Grid Automation}

Zhabelova and Vyatkin [83] proposed an agent implementation architecture for smart grid automation. Their work is aimed at developing practical automation architecture specific to the power system automation domain. The research facilitates migration of agent technology into the industrial practice.

The agent architecture is based on the industrial standards IEC 61850 [84] and IEC 61499 [85]. The first one provides a complete model of the power system automation domain, decomposing the domain into logical nodes (LNs) and defining a corresponding communication protocol. The agent is based on an IEC 61850 LN. An LN defines an agent's beliefs, purpose, or desire; it predefines the agent's intentions and possible actions. The IEC 61850 decomposition of the domain into automation functions gives a well-defined and structured domain model and helps with the agent identification. IEC 61499 is the foundation of the whole architecture. It employs current practice in the industrial automation while providing technologies required for modern automation systems, including the smart grid domain [86].

The proposed automation agent was applied in several projects, such as fault location isolation and supply restoration [83], intelligent fault management [87], intelligent energy management [88], and power balancing [89].

To simulate and to validate the developed agent-based solutions, a cosimulation framework was developed [22]. It allows system integration testing of the distributed control approach with the distributed power system model.

In all the mentioned projects, the agent system was developed and first simulated using IEC 61499 and then the system was directly deployed and executed on industrial hardware. The developed systems were tested on distributed PLCs (ADAM6650, Beckhoff CX1030-0120, and WAGO) and ARM boards.

\section{K. Further Projects and Activities With MAS Focus}

One of the first applications of agents in smart grids was reported and discussed by Gustavsson [90]. The load balancing task was modeled as a MAS with device agents (called HomeBots), service agents, and utility agent negotiating on a market via auctions. In another application, the agents are designed to monitor and control the office building's lights and temperature according to users' personal preferences.
Lanthaler and Gütl [91] present a distributed energy management system that reduces the energy wastage in camps of portable buildings. They are mainly used by mining companies in remote regions of Australia to temporarily inhabit the workers. The electricity for the camp is provided by a diesel generator, which implies high costs for the fuel and also high- $\mathrm{CO}_{2}$ emissions. The key issue is that the camp inhabitants waste a lot of energy especially by cooling down their rooms to $18^{\circ} \mathrm{C}$, even during periods of the day they are out of their homes. The proposed system for ubiquitous monitoring of energy consumption and control of home appliances consists of intelligent nodes and smart meters (installed at every house with central logging and control server infrastructure). The controller in the intelligent node automatically controls home appliances to reduce the energy waste without affecting the resident's comfort. The communication between the nodes and the central server is based on Internet protocols (e.g., TCP/IP and HTTP) and implemented in the form of RESTful API. The field trial evaluation was implemented in a remote housing site consisting of 1500 houses and was run for 7 months. By intelligent control of appliances, mainly the air conditioner which is the biggest energy consumer, the system was able to reduce the overall energy consumption by about $50 \%$.

An effort to develop a framework supporting the self-healing property of smart grids is presented in [92]. The infrastructure is based on decentralized principles with autonomous intelligent agents performing various tasks such as monitoring, performance enhancements, and control.

Belkacemi et al. [18] introduce the West Virginia SuperCircuit (WVSC) project that is part of the modern grid initiative aiming at demonstrating and testing new technologies to enable the deployment and implementation of smart grids. In this case, WVSC is used as a test bed to illustrate functionality of newly designed immune-based MAS. This system is inspired by natural systems such as ant colonies, bee colonies, or human immune cell system. The aim is to find analogies between the immune system and the MAS for power control to improve the robustness of distributed algorithms.

As already mentioned in Section II-C, the project is aimed at self-optimizing power grids through the application of MAS concepts [64]. The system architecture contains several agent types with different responsibilities (as represented for example in Fig. 3). The system agent is responsible for overall monitoring of voltage and frequency and has the ability of finding the best stable configuration of a power system by rerouting the power. It is done in cooperation with substation agents that in turn coordinate the functions of switch agents. The generator and load agents interact with the neighboring substation agent about the provided and needed power supply.

Karnouskos and de Holanda [27] present a simulator based on software agents that targets the modeling of the behavior of a Smart City. Energy generating and consuming devices, such as power stations, household appliances, and electric vehicles, or their aggregations such as households and cities, are simulated as JADE agents. There is a simple on/OFF strategy considered to achieve balancing of generation and consumption.

An integrated management system for smart grids based on agents is presented in [93]. There are various agents specialized 
in different tasks like data collection, status monitoring, configuration, and distribution. The semantic integration is ensured by the adoption of ontologies and rule-based inference engines.

The Advanced Research Institute of Virginia Tech presents the design and implementation of multiagent technology in the context of an intelligent, distributed, and autonomous power system [18]. The agents (i.e., control, DER, user, and database) are used to detect upstream outages and react accordingly to allow the micro grid to operate autonomously in an island.

Jahangiri et al. [94] introduce an agent-based distribution test feeder. Some of its components are modeled as interacting agents; for instance, a residence agent who determines the comfort-cost trade-offs, a plug-in electric vehicle agent trying to minimize energy costs considering feasible travel routes, and the photovoltaic agent who tries to maximize the harvested solar power with subject to panel surface area as well as weather conditions.

James et al. [95] report their pilot deployment of an agentbased distributed energy application called GridAgents in Australia. The application is developed in JADE. Agents controlling loads and generators are run on personal digital assistants (PDAs), whereas personal computers (PCs) are used for implementing more computationally intensive agent roles. Another pilot of a JADE-based agent application, described in Section IV-C, was deployed for a micro grid control used at the Greek Kythnos Island [43]. The agents control a micro grid with private and public photovoltaic generation, a battery bank, a diesel generation, as well as 12 residential houses.

The popularity of the JADE framework indicates the desire of researchers to test their agent-based algorithms in a distributed manner. JADE provides a good MAS prototyping and testing tool, but its heavy reliance on PC-based platforms hinders the migration of the developed MAS solution to industrial pilots. Also, not many authors place the importance on agent theory when designing the agents as well as agent-based solutions. There is a need for more practical and user friendly agent framework with formal theoretical foundations.

\section{Conclusion, Future Trends, And Research Activities}

\section{A. Advanced ICT Systems}

Smart grid is a technological paradigm being developed to satisfy the needs for considerable efficiency improvements in power generation and distribution processes. Recently, the vision of Internet of Energy (IoE) [96] has appeared in parallel to the concept of Internet of Things (IoT) [97]. In this idea, the objects and devices, used in people's everyday lives, are equipped with embedded intelligence and communication capabilities allowing them to be interconnected via the Internet. In the IoE vision, the grid will be based upon the intelligent communicating nodes acting on behalf of renewable resources, electric vehicles, home appliances, and smart meters, constituting the decentralized and service-oriented ecosystem.

1) Networked Devices: In order to implement this vision in smart grids, several hardware as well as ICT-based approaches have to be brought together. First of all, the use of small, low-cost, low-power, and resource constrained devices with pervasive computing capabilities is promoted as a key technological enabler for deploying a ubiquitous energy monitoring and control system [96]. The communication technologies have to be accordingly optimized due to hardware limitations of resource constrained devices. The influential standardization bodies such as Internet Engineering Task Force (IETF) and World Wide Web Consortium (W3C) are already working on variants of communication standards for resource constrained devices. It is for instance IPv6 over Low-Power Wireless Personal Area Networks (6LoWPAN) by IETF aiming at providing the resource constrained devices with the Internet connectivity through IPv6. As a matter of fact, the SmartHouse/SmartGrid project presented in Section IV-F reports on using the 6LoWPAN protocol within a smart home [26].

2) Proper Communication Approaches: Another IETF's initiative is called Constrained RESTful Environment with the main objective of developing the constrained application protocol (CoAP) providing the RESTful architectural style for constrained environments [98]. The W3C's efficient XML interchange (EFI) specification aims at reducing the size and parsing intricacy of XML resulting in the compression factors of up to $90 \%$ concerning device's memory use [96].

3) Open and Interoperable Standards: In general, flexible and open communication standards and infrastructures suitable to address smart grid specifics need to be developed [6], [7], [11], [12]. Major industrial actors are forming alliances, such as IPSO, Zigbee, or Homeplug, aiming at development of wired and wireless profiles suitable for smart grid. A profound attention has to be given to provide integrated ICT infrastructures that allow combining energy grid systems with other infrastructures at the urban level in order to fully explore the vision of Smart Cities. The exploration of the SOA concepts is expected to go down to the device level to further enhance the interoperability by offering the device functionality through services, while hiding the implementation details [25].

Application of standardized communication protocols is only one precondition achieving a system-wide interoperability. Another key factor is the capability of heterogeneous devices and software systems communicating in the smart grid environment to understand each other regardless of what different internal data models and formats they use. To address the issues of semantic interoperability various international standards are being developed. The IEC CIM approach (originally defined in IEC 61970) defines a unified vocabulary using unified modeling language (UML) notations that concerns physical aspects of energy management systems including SCADA. IEC 61968 extends CIM to deal with various aspects of distributed management systems, including outage management, planning, metering, asset management, and others. IEC 61850 promotes interoperability between devices within substations.

There are first attempts to make use of these standards in the smart grid domain. Like in case of [99], an open source-based energy management system using web-services or in [82] related to the ADDRESS approach where the XML content of messages is sent among web-services using CIM. Some researchers are proposing to combine CIM, semantic web-services, and OPC Unified Architecture (***OPC UA), which is the new platformindependent standard for industrial automation to deliver a 
semantic-aware ICT infrastructure, for both power transmission and distribution grids [100].

Moreover, the U.S.'s National Institute of Standards and Technology (NIST) aims at coordinating the development of protocols and model standards for achieving interoperability of smart grids [11]. To enable a more intelligent processing and reasoning about data conforming to these standards, their transcription into upper layer ontologies will be needed [9], [93].

4) Simulation, Visualization, and Validation Methods: Development of advanced simulation and visualization tools for the energy domain is another essential element supporting a fast roll-out of smart grid technologies. Basically, it is necessary to verify the solution before putting them in the ground as well as it is necessary to run the simulation in parallel with the real system (or slightly in advance) to be able to estimate what could happen if something goes wrong. Such a prediction is vital for instance to detect a threatening black out in its beginning in order to take fast actions to prevent it from spreading. Among most professional simulation tools, there is GridLAB-D developed by The U.S. Department of Energy at Pacific Northwest National Laboratory and PowerFactory by DigSilent. GridLAB-D ${ }^{9}$ is an open source suite of tools to build and manage power grid simulations, including agent-based modeling of real-time interactions of DERs, distributed automation and retail markets [101]. DigSilent's Power Factory is the hi-end tool for analysis of power systems dealing with power generation, transmission, and distribution. Another framework for simulation of active components, especially used in smart grids, is called mosaik [28].

5) Secure Infrastructure: Information and cyber security is another big challenge in smart grids. For instance, with the rollout of advanced metering infrastructures enabling to turn off the power remotely by utilities, there is an increased risk of attacks by cybercriminals which can cause severe damages by leaving large urban areas out of power. Several studies aimed at the identification of such security risks and review of existing security standards related to smart grids has been presented in [102]. It is obvious that substantial effort still needs to be devoted to provide standardized means guaranteeing secure communication, authentication, data confidentiality, and integrity. The matured security standards for web-services like webservices security (WWS) by OASIS can advocate the use of web-services as a unified communication infrastructure for smart grid systems.

\section{B. Open Research Questions}

Despite the growing awareness and the ongoing research activities in smart grids, there are still several open points which have to be covered in future research work. The most important points in the context of this review paper are as follows [103].

1) Advanced control functions: The ability to provide selfcorrective reconfiguration and restoration in the grid. Also handling the fluctuating behavior of DER devices as well as the upcoming integration of electric vehicles and gridscale storage devices is still an open point for further research. An integrated solution addressing advanced automation and control functions is missing. Such an

\footnotetext{
${ }^{9}$ Available: http://www.gridlabd.org
}

approach should be based on the MAS/SOA principles, guaranteeing interoperability, flexibility, and scalability.

2) Universal grids/hybrid grids: A lot of efforts are spent on the research of advanced functions for the electric energy infrastructure. A more comprehensive view on the whole energy system including other domains (e.g., gas, district heating, and water system) is a further and necessary step in order to achieve the vision of a green energy supply [104]. Approaches, concepts, methods, and standards form the electricity domain should be analyzed and applied to other energy-related domains. Especially, MAS/ SOA-based concepts would help to manage such complex energy systems.

3) Formal and model-driven design of automation systems: A common modeling language for automation and control applications is required. Together with a model-driven design support system, it would improve the development process of automation solutions in smart grids. It would increase the design and implementation productivity but also the code quality. It should be possible to design MASbased approaches with such an approach.

4) Advanced validation methods: Field tests are usually costly and time consuming. In order to test and validate control solutions for smart grids, proper validation methods have to be developed. A cosimulation-based approach seems to be suitable for testing advanced functions by covering the physical system, the communication network as well as the corresponding algorithms (including agents). Some parts of a complex smart grid can also be tested in a hardware-in-the-loop setup. The availability of suitable models covering all smart grid aspects as well as the coupling of the different simulators is still a broad field for further research.

5) Interoperability, scalability, and harmonization of standards: Developments related to MAS/SOA applied in smart grid context have to be aligned with domain standards. For example, standards like the IEC 61850 or the OPC UA should be used for agent-based communication [64].

\section{REFERENCES}

[1] M. Liserre, T. Sauter, and J. Hung, "Future energy systems: Integrating renewable energy sources into the smart power grid through industrial electronics," IEEE Ind. Electron. Mag., vol. 4, no. 1, pp. 18-37, Mar. 2010.

[2] G. Spagnuolo et al., "Renewable energy operation and conversion schemes: A summary of discussions during the seminar on renewable energy systems," IEEE Ind. Electron. Mag., vol. 4, no. 1, pp. 38-51, Mar. 2010

[3] H. Farhangi, "The path of the smart grid," IEEE Power Energy Mag., vol. 8, no. 1, pp. 18-28, Jan./Feb. 2010.

[4] IEA, Technology Roadmap Smart Grids. (2011). International Energy Agency (IEA), Paris, France, Tech. Rep. [Online]. Available: http://www. iea.org

[5] C. Cecati, G. Hancke, P. Palensky, P. Siano, and X. Yu, "Guest editorial special section on information technologies in smart grids," IEEE Trans. Ind. Informat., vol. 9, no. 3, pp. 1380-1383, Aug. 2013.

[6] V. C. Gungor et al., "A survey on smart grid potential applications and communication requirements," IEEE Trans. Ind. Informat., vol. 9, no. 1, pp. 28-42, Feb. 2013.

[7] V. Gungor et al., "Smart grid technologies: Communication technologies and standards," IEEE Trans. Ind. Informat., vol. 7, no. 4, pp. 529-539, Nov. 2011 
[8] A. Sabbah, A. El-Mougy, and M. Ibnkahla, "A survey of networking challenges and routing protocols in smart grids," IEEE Trans. Ind. Informat., vol. 10, no. 1, pp. 210-221, Feb. 2014.

[9] E. M. Davidson, V. M. Catterson, and S. D. J. McArthur, "The role of intelligent systems in delivering the smart grid," in Proc. Power Energy Soc. Gen. Meet., 2010, pp. 1-6.

[10] J. K. Kok, C. J. Warmer, and I. G. Kamphuis, "PowerMatcher: Multiagent control in the electricity infrastructure," in Proc. 4th Int. Joint Conf. Auton. Agents Multiagent Syst., 2005, pp. 75-82.

[11] NIST, NIST framework and roadmap for Smart Grid interoperability standards. (2010). National Inst. Std. Technol. (NIST), U.S. Dept. Commerce, Gaithersburg, MD, USA, Tech. Rep. NIST Publication 1108. [Online]. Available: http://www.nist.gov

[12] SMB Smart Grid Strategic Group (SG3). (2010). IEC Smart Grid standardization roadmap. Int. Electrotech. Commission (IEC), Geneva, Switzerland, Tech. Rep. Ed. 1.0 [Online]. Available: http://www.iec.ch

[13] X. Yu, C. Cecati, T. Dillon, and M. Simões, "The new frontier of smart grids," IEEE Ind. Electron. Mag., vol. 5, no. 3, pp. 49-63, Sep. 2011.

[14] Y. Cao, W. Yu, W. Ren, and G. Chen, "An overview of recent progress in the study of distributed multi-agent coordination," IEEE Trans. Ind. Informat., vol. 9, no. 1, pp. 427-438, Feb. 2013.

[15] P. Leitao, V. Marik, and P. Vrba, "Past, present, and future of industrial agent applications," IEEE Trans. Ind. Informat., vol. 9, no. 4, pp. 2360-2372, Nov. 2013.

[16] M. Pěchouček and V. Mařík, "Industrial deployment of multi-agent technologies: Review and selected case studies," Auton. Agents MultiAgent Syst., vol. 17, no. 3, pp. 397-431, 2008.

[17] P. Vrba et al., "Rockwell automation's holonic and multi-agent control systems compendium," IEEE Trans. Syst. Man, Cybern. C., Appl. Rev., vol. 41, no. 1, pp. 14-30, Jan. 2011.

[18] R. Belkacemi, A. Feliachi, M. A. Choudhry, and J. E. Saymansky, "Multi-Agent systems hardware development and deployment for smart grid control applications," in Proc. Power Energy Soc. Gen. Meet., 2011, pp. $1-8$.

[19] V. Loia, A. Vaccaro, and K. Vaisakh, "A self-organizing architecture based on cooperative fuzzy agents for smart grid voltage control," IEEE Trans. Ind. Informat., vol. 9, no. 3, pp. 1415-1422, Aug. 2013.

[20] S. McArthur et al., "Multi-agent systems for power engineering applications-Part I: Concepts, approaches, and technical challenges," IEEE Trans. Power Syst., vol. 22, no. 4, pp. 1743-1752, Nov. 2007.

[21] A. L. Dimeas and N. D. Hatziargyriou, "Design of a MAS for an Island System," in Proc. Int. Conf. Intell. Syst. Appl. Power Syst., 2007, pp. 1-3.

[22] C. Yang, G. Zhabelova, C.-W. Yang, and V. Vyatkin, "Cosimulation environment for event-driven distributed controls of smart grid," IEEE Trans. Ind. Informat., vol. 9, no. 3, pp. 1423-1435, Aug. 2013.

[23] F. Jammes and H. Smit, "Service-oriented paradigms in industrial automation," IEEE Trans. Ind. Informat., vol. 1, no. 1, pp. 62-70, Feb. 2005.

[24] J. Puttonen, A. Lobov, and J. L. Martinez Lastra, "Semantics-based composition of factory automation processes encapsulated by web services," IEEE Trans. Ind. Informat., vol. 9, no. 4, pp. 2349-2359, Nov. 2013.

[25] S. Karnouskos, "Cyber-physical systems in the SmartGrid," in Proc. 9th IEEE Int. Conf. Ind. Informat. (INDIN), 2011, pp. 20-23.

[26] S. Karnouskos et al., "Monitoring and control for energy efficiency in the smart house," in Energy-Efficient Computing and Networking. Berlin, Germany: Springer-Verlag, 2011.

[27] S. Karnouskos and T. N. de Holanda, "Simulation of a smart grid city with software agents," in Proc. 3rd UKSim Eur. Symp. Comput. Model. Simul. (EMS), 2009, pp. 424-429.

[28] S. Schutte, S. Scherfke, and M. Troschel, "Mosaik: A framework for modular simulation of active components in Smart Grids," in Proc. IEEE 1st Int. Workshop Smart Grid Model. Simul., 2011, pp. 55-60.

[29] European Commission, European SmartGrids technology platform: Vision and strategy for Europe's electricity networks of the future. (2006). Directorate-General for Research-Sustainable Energy Systems, European Commission, Brussels, Belgium, Tech. Rep. EUR 22040 [Online]. Available: http://cordis.europa.eu

[30] N. Hatziargyriou, "Smart agent technology to help DER integrate markets: Experiments in Greece," in Proc. 3rd Int. Conf. Integr. Renew. Distrib. Energy Resour., Nice, France, 2010.

[31] International Energy Agency. (2012). World Energy Outlook 2012 [Online]. Available: http://www.iea.org

[32] P. Palensky and D. Dietrich, "Demand side management: Demand response, intelligent energy systems, and smart loads," IEEE Trans. Ind. Informat., vol. 7, no. 3, pp. 381-388, Aug. 2011.

[33] J. A. P. Lopes, F. J. Soares, and P. M. R. Almeida, "Integration of electric vehicles in the electric power system," Proc. IEEE, vol. 99, no. 1, pp. 168-183, Jan. 2011.
[34] F. Kennel, D. Gorges, and S. Liu, "Energy management for smart grids with electric vehicles based on hierarchical MPC," IEEE Trans. Ind. Informat., vol. 9, no. 3, pp. 1528-1537, Aug. 2013.

[35] A. Lugmaier, H. Brunner, B. Bletterie, F. Kupzog, and A. Abart, "Intelligent distribution grids in respect of a growing share of distributed generation," in Proc. 19th Int. Conf. Electr. Distrib., 2007, pp. 1-4.

[36] J. Mutale, "Benefits of active management of distribution networks with distributed generation," in Proc. IEEE Power Syst. Conf. Expo., 2006, pp. 601-606.

[37] W. Prüggler, F. Kupzog, B. Bletterie, and B. Helfried, "Active grid integration of distributed generation utilizing existing infrastructure more efficiently-An Austrian case study," in Proc. 5th Int. Conf. Eur. Electr. Market, 2008, pp. 1-6.

[38] G. J. Schaeffer and H. Akkermand. (2006). CRISP Final Summary Report [Online]. Available: http://www.crisp.ecn.nl/documents.html

[39] J. A. P. Lopes, N. Hatziargyriou, J. Mutale, P. Djapic, and N. Jenkins, "Integrating distributed generation into electric power systems: A review of drivers, challenges and opportunities," Electr. Power Syst. Res., vol. 77, pp. 1189-1203, Jul. 2007.

[40] R. A. Walling, R. Saint, R. C. Dugan, J. Burke, and A. L. Kojovic, "Summary of distributed resources impact on power delivery systems," IEEE Trans. Power Del., vol. 23, no. 3, pp. 1636-1644, 2008

[41] J. K. Kok, "Multi-agent coordination in the electricity grid, from concept towards market introduction," in Proc. 9th Int. Conf. Auton. Agents Multiagent Syst.: Ind. Track, 2010, pp. 1681-1688.

[42] A. L. Dimeas and N. D. Hatziargyriou, "Agent based control of virtual power plants," in Proc. Int. Conf. Intell. Syst. Appl. Power Syst., 2007, pp. 1-6.

[43] S. J. Chatzivasiliadis, N. D. Hatziargyriou, and A. L. Dimeas, "Development of an agent based intelligent control system for microgrids," in Proc. Power Energy Soc. Gen. Meet.-Convers. Del. Elect. Energy 21st Century, 2008, pp. 1-6.

[44] D. Issicaba, N. J. Gil, and J. A. Peas Lopes, "Islanding operation of active distribution grids using an agent-based architecture," in Proc. IEEE PES Innovat. Smart Grid Technol. Conf. Eur., 2010, pp. 1-8.

[45] I. J. Balaguer, U. Supatti, Q. Lei, N.-S. Choi, and F. Z. Peng, "Intelligent control for intentional islanding operation of microgrids," in Proc. IEEE Int. Conf. Sustain. Energy Technol., 2008, pp. 898-903.

[46] V. Calderaro, V. Lattarulo, A. Piccolo, and P. Siano "Optimal switch placement by alliance algorithm for improving microgrids reliability," IEEE Trans. Ind. Informat., vol. 8 no. 4, pp. 925-934, Nov. 2012.

[47] A. Viehweider, H. Schichl, D. B. de Castro, S. Henein, and D. Schwabeneder, "Smart robust voltage control for distribution networks using interval arithmetic and state machine concepts," in Proc. IEEE PES Innovat. Smart Grid Technol. Conf. Eur., 2010, pp. 1-8.

[48] F. Kupzog, H. Brunner, W. Pruggler, T. Pfajfar, and A. Lugmaier, "DG DemoNet-concept - A new algorithm for active distribution grid operation facilitating high DG penetration," in Proc. 5th IEEE Int. Conf. Ind. Informat., 2007, pp. 1197-1202.

[49] M. A. M. Manaz, D. B. W. Abeywardana, M. G. C. P. Mediwaththe, K. M. Liyanage, and A. Yokoyama, "Active and reactive power balancing of ubiquitous power networks considering voltage constraints," in Proc. 7th IEEE Int. Conf. Ind. Inf. Syst., 2012, pp. 1-6.

[50] P. Mitra, G. K. Venayagamoorthy, and K. A. Corzine, "SmartPark as a virtual STATCOM," IEEE Trans. Smart Grid, vol. 2, no. 3, pp. 445-455, Sep. 2011.

[51] J. Deuse, "Profitable integration of DER: From market to new regulation principles," Keynote presentation at 3rd Int. Conf. Integr. Renew. Distrib. Energy Resour., Nice, France, 2010.

[52] J. M. Jorgensen, S. H. Sorensen, K. Behnke, and P. B. Eriksen, "EcoGrid EU-A prototype for European Smart Grids," in Proc. IEEE Power Energy Soc. Gen. Meet., 2011, pp. 1-7.

[53] G. Peppink et al., "INTEGRAL: ICT-platform based distributed control in electricity grids with a large share of distributed energy resources and renewable energy sources," in Energy-Efficient Computing and Networking, N. Hatziargyriou, A. Dimeas, T. Tomtsi, and A. Weidlich, Eds. Berlin, Germany: Springer-Verlag, 2011, pp. 215-224.

[54] R. Palma-Behnke, J. L. A. Cerda, L. S. Vargas, and A. Jofre, “A distribution company energy acquisition market model with integration of distributed generation and load curtailment options," IEEE Trans. Power Syst., vol. 20, no. 4, pp. 1718-1727, Nov. 2005.

[55] A. J. D. Rathnayaka, V. M. Potdar, T. S. Dillon, O. K. Hussain, and E. Chang, "A methodology to find influential prosumers in prosumer community groups," IEEE Trans. Ind. Informat., vol. 10, no. 1, pp. 706-713, Feb. 2014. 
[56] H. Pidd. (2012). India blackouts leave 700 million without power. The Guardian [Online]. Available: http://www.theguardian.com/world/2012/ jul/31/india-blackout-electricity-power-cuts

[57] G. Rigatos, P. Siano, and A. Piccolo, "Neural network-based approach for early detection of cascading events in electric power systems," IET Gener. Transm. Distrib., vol. 3, no. 7, pp. 650-665, 2009.

[58] H. Tram, "Technical and operation considerations in using Smart Metering for outage management," in Proc. IEEE PES Transm. Distrib. Conf. Expo., 2008, pp. 1-3.

[59] E. Bjerkan, "Efficient fault management using remote fault indicators," in Proc. 20th Int. Conf. Exhib. Electr. Distrib. Part 1, 2009, pp. 1-4.

[60] J. O. Wambauch, "Smart metering for the distribution control room," in Proc. 22nd Int. Conf. Exhib. Electr. Distrib., 2013, pp. 1-4.

[61] S. T. Cha, A. Saleem, W. Qiuwei, and J. Ostergaard, "Agent based particle swarm optimization for load frequency control of distribution grid," in Proc. 47th Int. Univ. Power Eng. Conf., 2012, pp. 1-6.

[62] J. DongLi, M. XiaoLi, and S. XiaoHui, "Study on technology system of self-healing control in smart distribution grid," in Proc. Int. Conf. Adv. Power Syst. Autom. Protect., 2011, pp. 26-30.

[63] S. Bou Ghosh et al., "Agent-oriented designs for a self healing smart grid," in Proc. 1st IEEE Int. Conf. Smart Grid Commun., 2010, pp. $461-466$.

[64] M. Merdan, W. Lepuschitz, T. Strasser, and F. Andren, "Multi-agent system for self-optimizing power distribution grids," in Proc. 5th Int. Conf. Autom. Robot. Appl., 2011, pp. 312-317.

[65] M. Wooldridge, An Introduction to Multi-Agent Systems. Hoboken, NJ, USA: Wiley, 2002.

[66] P. Leitão, "Agent-based distributed manufacturing control: A state-of-theart survey," Eng. Appl. Artif. Intell., vol. 22, no. 7, pp. 979-991, 2009.

[67] J. M. Mendes, P. Leitão, F. Restivo, and A. W. Colombo, "Serviceoriented agents for collaborative industrial automation and production systems," in Proc. 4th Int. Conf. Ind. Appl. Holonic Multi-Agent Syst. (HoloMAS), 2009, pp. 13-24.

[68] L. Guo, M. Ghanem, V. Curcin, and N. Azam, "Intelligent agents in the service-oriented world - An industrial experience report," in Proc. IEEE/ WIC/ACM Int. Joint Conf. Web Intell. Intell. Agent Technol. (WI-IAT'09), Milan, Italy, Sept. 15-18, 2009, pp. 693-696.

[69] V. V. Herrera et al., "Integration of multi-agent systems and serviceoriented architecture for industrial automation," in Proc. 6th IEEE Int. Conf. Ind. Informat. (INDIN'08), Daejeon, Korea, Jul. 13-16, 2008, pp. $768-773$.

[70] V. Gungor et al., "Smart grid and smart homes: Key players and pilot projects," IEEE Ind. Electron. Mag., vol. 6, no. 4, pp. 18-34, Dec. 2012

[71] P. Leitao, P. Vrba, and T. Strasser, "Multi-agent systems as automation platform for intelligent energy systems," in Proc. 39th Аnnu. Conf. IEEE Ind. Electron. Soc., Vienna, Austria, Nov. 10-13, 2013, pp. 66-71.

[72] V. M. Catterson, E. M. Davidson, and S. D. J. McArthur, "Issues in integrating existing multi-agent systems for power engineering applications," in Proc. 13th Int. Conf. Intell. Syst. Appl. Power Syst., 2005, pp. 1-6.

[73] M. Pipattanasomporn, H. Feroze, and S. Rahman, "Multi-agent systems in a distributed smart grid: Design and implementation," in Proc. IEEE/PES Power Syst. Conf. Expo., 2009, pp. 1-8.

[74] Z. Zhang and M.-Y. Chow, "Incremental cost consensus algorithm in a smart grid environment," in Proc. IEEE Power Energy Soc. Gen. Meet., 2011, pp. $1-6$

[75] K. de Geus et al., "A distributed model for electrical substation automation based on multi-agents technology," in Proc. Int. Conf. Intell. Modell. Control Autom., Int. Conf. Intell. Agents Web Technol. Internet Commerce (CIMCA-IAWTIC), 2006, p. 256.

[76] A. Payne, J. Jarvis, D. Jarvis, and N. Higgins, "A holonic architecture for feeder restoration," in Proc. Annu. Conf. IEEE Ind. Electron. Soc. (IECON), Melbourne, Australia, 2011.

[77] J. A. Hossack, J. Menal, S. D. J. McArthur, and J. R. McDonald, "Multiagent architecture for protection engineering diagnostic assistance," IEEE Trans. Power Syst., vol. 18, no. 2, pp. 639-647, May 2003.

[78] K. Kok et al., "Agent-based electricity balancing with distributed energy resources, A multiperspective case study," in Proc. 41st Hawaii Int. Conf. Syst. Sci., Waikoloa, HI, USA, 2008, pp. 1-10.

[79] Foundation for Intelligent Physical Agents. (2014). Agent Communication Language Specifications [Online]. Available: http://www.fipa.org/ repository/aclspecs.html

[80] P. Romanos, N. Hatziargyriou, J. Schmid, and J. Mutale, "Single agents in smart grids," in Proc. 7th Medit. Conf. Exhib. Power Gener. Transm. Distrib. Energy Convers., 2010, pp. 1-9.
[81] R. Belhomme, R. Cerero, G. Valtorta, and P. Eyrolles, "The ADDRESS project: Developing active demand in smart power systems integrating renewables," in Proc. IEEE Power Energy Soc. Gen. Meet., 2011, pp. 1-8.

[82] C. Effantin, T. Kostic, and E. Lambert. (2011). ADDRESS Project Deliverable D4.1: Documentation of Software Architecture and Encoding in UML Including Compiled Software with API Description-Models for Interoperable Information Exchanges Between ADDRESS Actors [Online]. Available: http://www.addressfp7.org

[83] G. Zhabelova and V. Vyatkin, "Multiagent Smart Grid automation architecture based on IEC 61850/61499 intelligent logical nodes," IEEE Trans. Ind. Electron., vol. 59, no. 5, pp. 2351-2362, May 2012.

[84] International Electrotechnical Commission, "IEC 61850 Communication networks and systems for power utility automation," Geneva, Switzerland, Tech. Rep. IEC 61850, 2009

[85] International Electrotechnical Commission, "IEC 61499-1 Function blocks-Architecture," Geneva, Switzerland, Tech. Rep. IEC 61499, 2005.

[86] G. Zhabelova, S. Patil, C.-W. Yang, and V. Vyatkin, "Smart Grid applications with IEC 61499 reference architecture," in Proc. 11th IEEE Int. Conf. Ind. Informat. (INDIN), 2013, pp. 458-463.

[87] G. Zhabelova, V. Vyatkin, and N. Nair, "Standard-based engineering and distributed execution framework for intelligent fault management for FREEDM system," in Proc. 37th Annu. Conf. IEEE Ind. Electron. Soc., Melbourne, Australia, 2011, pp. 2724-2729.

[88] G. Zhabelova, Z. Zhang, V. Vyatkin, and M.-Y. Chow, "Agent-based distributed consensus algorithm for decentralized economic dispatch in Smart Grid," in Proc. 39th Annu. Conf. IEEE Ind. Electron. Soc., Vienna, Austria, 2013, pp. 1968-1973.

[89] S. Patil, V. Vyatkin, and B. McMillin, "Implementation of FREEDM Smart Grid distributed load balancing using IEC 61499 function blocks," in Proc. 39th Annu. Conf. IEEE Ind. Electron. Soc., 2013, pp. 8154-8159.

[90] R. Gustavsson, "Agents with power," Commun. ACM, vol. 42, pp. 41-47, 1999.

[91] M. Lanthaler and C. Gütl, "A Web of Things to reduce energy wastage," in Proc. 10th IEEE Int. Conf. Ind. Informat., 2012, pp. 1050-1055.

[92] K. Moslehi et al., "Framework for a self-healing power grid," in Proc. IEEE Power Eng. Soc. Gen. Meet., 2005, vol. 3, pp. 1-8.

[93] A. P. Garcia, J. Oliver, and D. Gosch, "An intelligent agentbased distributed architecture for Smart-Grid integrated network management," in Proc. IEEE 35th Conf. Local Comput. Netw., 2010, pp. 1013-1018.

[94] P. Jahangiri, W. Di, L. Wanning, D. C. Aliprantis, and L. Tesfatsion, "Development of an agent-based distribution test feeder with smart-grid functionality," in Proc. IEEE Power Energy Soc. Gen. Meet., 2012, pp. 1-7.

[95] G. James, D. Cohen, R. Dodier, G. Platt, and D. Palemer, "A deployed multi-agent framework for distributed energy applications," in Proc. 5th Int. Joint Conf. Auton. Agents Multiagent Syst., 2006, pp. 676-678.

[96] N. Bui, A. P. Castellani, P. Casari, and M. Zorzi, "The internet of energy: A web-enabled smart grid system," IEEE Netw., vol. 26, no. 4, pp. 39-45, Jul./Aug. 2012.

[97] J. Gubbi, R. Buyya, S. Marusic, and M. Palaniswami, "Internet of Things (IoT): A vision, architectural elements, and future directions," Future Gener. Comput. Syst., vol. 29, no. 7, pp. 1645-1660, 2013.

[98] C. Bormann, A. P. Castellani, and Z. Shelby, "CoAP: An application protocol for billions of tiny internet nodes," IEEE Internet Comput., vol. 16, no. 2, pp. 62-67, Mar./Apr. 2012

[99] A. Mercurio, A. Di Giorgio, and P. Cioci, "Open-source implementation of monitoring and controlling services for EMS/SCADA systems by means of web services-IEC 61850 and IEC 61970 standards," IEEE Trans. Power Del., vol. 24, no. 3, pp. 1148-1153, Jul. 2009.

[100] S. Rohjans, M. Uslar, and H. J. Appelrath, "OPC UA and CIM: Semantics for the smart grid," in Proc. IEEE PES Transm. Distrib. Conf. Expo., 2010, pp. $1-8$.

[101] D. P. Chassin, K. Schneider, and C. Gerkensmeyer, "GridLAB-D: An open-source power systems modeling and simulation environment," in Proc. IEEE/PES Transm. Distrib. Conf. Expo., 2008, pp. 1-5.

[102] W. YuFei, Z. Bo, L. WeiMin, and Z. Tao, "Smart grid information security -A research on standards," in Proc. Int. Conf. Adv. Power Syst. Autom. Protect., 2011, pp. 1188-1194.

[103] T. Strasser, F. Andrén, M. Merdan, and A. Prostejovsky, "Review of trends and challenges in smart grids: An automation point of view," in Industrial Applications of Holonic and Multi-Agent Systems. Berlin, Germany: Springer-Verlag, 2013, pp. 1-12.

[104] C. Cecati, C. Citro, A. Piccolo, and P. Siano, "Smart operation of wind turbines and diesel generators according to economic criteria," IEEE Trans. Ind. Electron., vol. 58, no. 10, pp. 4514-4525, Oct. 2011. 


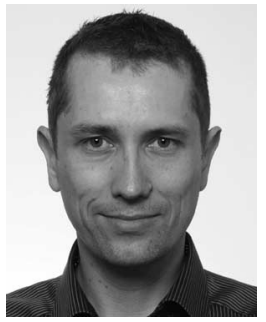

Pavel Vrba (M'05-SM'14) received the Ph.D. degree in applied sciences and informatics, cybernetics from the University of West Bohemia, Pilsen, Czech Republic, in 2001

$\mathrm{He}$ is an Assistant Professor with the Department of Cybernetics, Czech Technical University in Prague, Prague, Czech Republic, where he leads the Intelligent Systems Group. He was with the Rockwell Automation Research Center, Prague, from 2001 to 2012, where he led the Distributed Intelligent Control Lab from 2005 to 2012. He has published more than 80 conference papers, journal articles, and book chapters related to his research area, and has five filed and one pending U.S. patents. His research interests include intelligent industrial control systems; holonic and multiagent systems; cyberphysical systems; systems of systems; Internet of Things; semantic technologies and ontologies; service-oriented architectures; and agent-based modeling, simulation, and visualization.

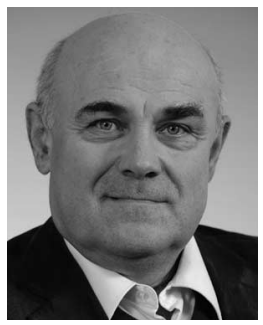

Vladimír Mařík (M'95-SM'13) received the Ph.D. degree in cybernetics from the Czech Technical University (CTU), Prague, Czech Republic, in 1979.

He was appointed as a Full Professor at CTU in 1990 , and is a Director with the newly established Czech Institute for Informatics, Robotics, and Cybernetics (CIIRC). Earlier, he acted as Head of the Department of Cybernetics, CTU, from 1999 to 2013, and as a Founder and Director with the Rockwell Automation Research Center, Prague, from 1992 to 2009. His research interests include artificial intelligence, multiagent systems, knowledge-based systems, soft-computing, production planning, and scheduling applications.

Mr. Mařík served as the Editor-in-Chief of the IEEE TRAnsactions on Systems, Man, and Cybernetics-Part C: Applications and Review from 2005 to 2013 . He is currently serving as a Vice-President of the IEEE Systems, Man, and Cybernetics Society.

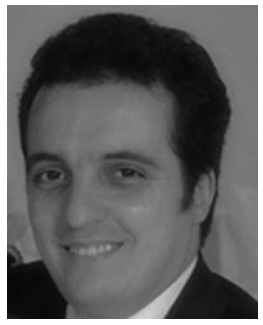

Pierluigi Siano (M'09-SM'14) received the M.Sc. degree in electronic engineering and the Ph.D. degree in information and electrical engineering from the University of Salerno, Salerno, Italy, in 2001 and 2006, respectively.

$\mathrm{He}$ is an Aggregate Professor of Electrical Energy Engineering with the Department of Industrial Engineering, University of Salerno. In these fields, he has coauthored more than 160 papers, including more than 70 in international journals. His research interests include the integration of distributed energy resources in efficient and smart distribution systems, and on the application of soft computing methodologies to planning and management of power systems.

Dr. Siano is an Associate Editor with the IEEE Transactions on Industrial INFORMATICS, and is a Member of the editorial board of more than twenty international journals in the field of power systems and smart grids. He is the Vice-Chair of the Technical Committee on smart grids and a member of the Technical Committee on Renewable Energy Systems of the IEEE Industrial Electronics Society (IES). He was a Special Sessions Co-Chair of the IEEE International Symposium on Industrial Electronics (ISIE)'10, and has been a Guest Editor of many special sections in IEEE TRANSACtions on IndustriaL Electronics and IEEE Transactions on Industrial INFormatics.

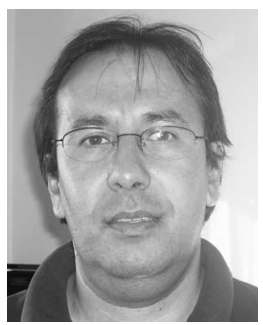

Paulo Leitão (M’98-SM’08) received the M.Sc. and $\mathrm{Ph} . \mathrm{D}$. degrees in electrical and computer engineering from the University of Porto, Porto, Portugal, in 1997 and 2004, respectively.

He is a Coordinator (Associate) Professor with the Polytechnic Institute of Bragança, Bragança, Italy, and currently, the Head of the Department of Electrical Engineering and a member of the Artificial Intelligence and Computer Science Laboratory (LIACC). He has published more than 130 papers in high-ranked international scientific journals and conference proceedings. He is co-author of three patents. His research interests include industrial informatics, collaborative factory automation, reconfigurable production systems, intelligent supervisory control, agent-based and holonic control, and bioinspiration engineering.

Dr. Leitão served as General Co-Chair of several international conferences, including the International Federation of Automatic Control Intelligent Manufacturing Systems (IFAC IMS)' 10 and the International Conference on Industrial Applications of Holonic and Multi-Agent Systems (HoloMAS)'11. Currently, he is the Chair of the IEEE Industrial Electronics Society Technical Committee on Industrial Agents.

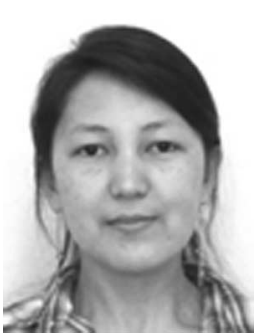

Gulnara Zhabelova received the Diploma degree in robotics and mechatronics, and the M.E. degree in automation and control from Karaganda State Technical University, Karaganda, Kazakhstan, in 2006 and 2008, respectively, and the M.E. degree in computer systems and the Ph.D. degree in electrical and electronics engineering from the University of Auckland, Auckland, New Zealand, in 2009 and 2013, respectively.

Currently, she is a Research Engineer with the Luleå University of Technology, Luleå, Sweden. Previously, she was with the Industrial Informatics Lab, University of Auckland, from 2009 to 2013. Her research interests include agent technology and its formal definition, theory, and application in wide practical domains, including automation and control; protection in energy generation, transmission, distribution, and consumption; building automation; demand side management; advanced metering infrastructure (AMI); energy markets; and policies.

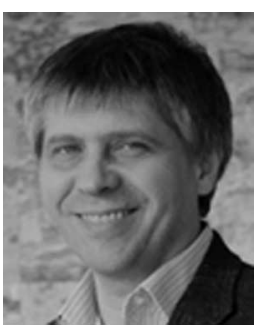

Valeriy Vyatkin (M'03-SM'04) received the Ph.D degree from the State University of Radio Engineering, Taganrog, Russia, in 1992.

$\mathrm{He}$ is on joint appointment as Chaired Professor (Åmnes Professor) of Dependable Computation and Communication Systems, Luleå University of Technology, Luleå, Sweden, and Professor of Information and Computer Engineering in Automation at Aalto University, Helsinki, Finland. Previously, he was a Visiting Scholar at Cambridge University, Cambridge, U.K., and has permanent academic appointments with the University of Auckland, Auckland, New Zealand; Martin Luther University of Halle-Wittenberg, Halle, Germany; Taganrog State University of Radio Engineering, Taganrog, Russia; and a Postdoc with the Nagoya Institute of Technology, Nagoya, Japan. His research interests include dependable distributed automation and industrial informatics; software engineering for industrial automation systems; and distributed architectures and multiagent systems applied in various industry sectors, including smart grid, material handling, building management systems, and reconfigurable manufacturing.

Dr. Vyatkin was awarded the Andrew P. Sage Award for the best IEEE Transactions paper in 2012 .

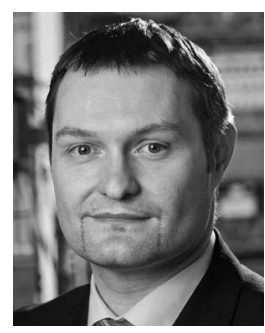

Thomas Strasser (M'09-SM'13) received the Ph.D. degree in mechanical engineering with a focus on automation and control theory from Vienna University of Technology, Vienna, Austria, in 2003.

$\mathrm{He}$ is Senior Scientist with the Austrian Institute of Technology (AIT), Vienna, in the domain of smart grids with focus on power utility automation. He was working as a Senior Researcher with PROFACTOR Research, Steyr, Austria, in the field of reconfigurable automation for six years.

Dr. Strasser a member of the International Electrotechnical Commission-Subcommittee 65B/Working Group 15-Function Blocks (IEC SC65B/WG15) maintaining the IEC 61499 standard. In addition, he is Senior Member of the IEEE System, Man, and Cybernetics Society (SMC) and the IEEE Power and Energy Society (PES). 